\title{
Spatial Map Learning with Self-Organizing Adaptive Recurrent Incremental Network
}

\author{
Wei Hong Chin*, Naoyuki Kubota*, Chu Kiong Loo ${ }^{\dagger}$, Zhaojie Ju${ }^{\ddagger}$ and Honghai Liu \\ ${ }^{*}$ Faculty of Systems Design, Tokyo Metropolitan University, Hino, Japan \\ Email: chin-weihong@ed.tmu.ac.jp, kubota@tmu.ac.jp \\ ${ }^{\dagger}$ Faculty of Computer Science \& Technology, University of Malaya, Malaysia \\ Email: ckloo.um@um.edu.my \\ $\ddagger$ University of Portsmouth, Portsmouth, UK \\ Email: \{zhaojie.ju, honghai.liu\}@ port.ac.uk
}

\begin{abstract}
Biological information inspires the advancement of a navigational mechanism for autonomous robots to help people explore and map real-world environments. However, the robot's ability to constantly acquire environmental information in realworld, dynamic environments has remained a challenge for many years. In this paper, we propose a self-organizing adaptive recurrent incremental network that models human episodic memory to learn spatiotemporal representations from novel sensory data. The proposed method termed as SOARIN consists of two main learning process that is active learning and episodic memory playback. For active learning (robot exploration), SOARIN quickly learns and adapts incoming novel sensory data as episodic neurons via competitive Hebbian Learning. Episodic neurons are connecting with each other and gradually forms a spatial map that can be used for robot localization. Episodic memory playback is triggered whenever the robot is in an inactive mode (charging or hibernating). During playback, SOARIN gradually integrates knowledge and experience into more consolidate spatial map structures that can overcome the catastrophic forgetting. The proposed method is analyzed and evaluated in term of map learning and localization through a series of real robot experiments in real-world indoor environments.
\end{abstract}

Keywords-Episodic Memory, Spatial Map, SLAM, Navigation.

\section{INTRODUCTION}

One of the essential features of common living locomotive organisms is their capability to traverse their daily environment with life-critical tasks. For example, rats can learn to visit or avoid places of food that they have visited and squirrels are excellent at rediscovering places of food that they have previously hidden. Many animals escape to a shelter that previously visited if they are undergoing an urgent threat such as a bear that escapes to a cave for hibernation to preserve energy during the winter season. It is a specious hypothesis that living organisms should have a cognitive mechanism to represent their environment as a collection of important regions, such as nest locations and food places. They can recall these regions and utilize relations between these regions when necessary to perform navigation tasks [1].

The capability of an autonomous mobile robot to represent its environment as a spatial map and to determine its position concurrently has been widely analyzed in the robotics society.
The process is termed as SLAM (simultaneous location and mapping) and several state-of-the-art have been introduced that work remarkably well [2], [3], [4]. However, these methods are essentially applied in moderately static indoor spaces and for a short period of time.

The majority of autonomous methods rely on two types of sensor data for SLAM operation. The first type of sensing data is collected from external signals using active sensors and passive sensors. Radar, laser or sonar-based obstacle detectors and range finders are active sensors whereas cameras, Kinects and microphones are passive sensors. The second type of data comes from proprioceptive indications such as wheel odometry, compass, and inertial measurements. With the external indicators, the localization estimation can be accomplished adequately as long as the environment remains relatively consistent. The proprioceptive indications are normally susceptible to failure due to the environment changes either because of natural phenomena such as differences in lighting conditions or weather, or because of human-made changes such as relocation of furniture, or previously visited routes become inaccessible. In contrast, proprioceptive sensors are superior to external signals, therefore, these sensors able to provide collateral information when autonomous agents receiving ambiguous external signals.

Biological methods do not appear to experience enormously from the above-mentioned deficiencies in artificial navigation. For example, rats are able to explore, search, and travel in large and dynamic environments for a long period of time. They can adapt to the changes of environment quickly, for instance, searching new ways if a previously visited route is unavailable or choosing potential shortcuts when new access spots are available. Therefore, a number of computer goal-oriented navigation systems were introduced to partially emulate how the brain could represent space and apply these representations for navigation tasks.

One of the biologically-inspired proposed methods is RatSLAM [5]. The approach represents the environment as a set of pose cells and each pose cell is linked to a view cell. RatSLAM was successfully implemented in small and large environments for spatial mapping but the framework does not handle targetoriented navigation. Erdem and Hasselmo [6] proposed a 
biologically inspired computational model for goal-oriented navigation. In this model, the environment is represented as several grid cells with different scale and spacing and gradually converge into one place cell. The model gradually recruits new place cells to encode autonomous agent's current location when the agent meets notable location during exploration. Each place cell has a reward cell and the lateral weight of the connection between two reward cells is equivalent to the time between the autonomous agent's successive visits to the reward cells. With the lateral connections, autonomous agents able a way to navigate to the goal location from its starting location.

However, the above-mentioned methods focus on emulating place cells and grid cells for spatial map building. Humans seem to accommodate themselves better in complex environments and recall past experiences to perform tasks at the same time generate new experience and skills. These significant behaviors usually develop from experiences that rely on learning. Likewise, the assumption is that experience also implies for robots [7]. Thus, the learned experiences can be integrated into a spatial map so that robots can freely observe and navigate in any environment. Memory is a fundamental perspective for the acquisition of experience. Memory is essential for the understanding, learning, and cognition of the interactions of robots in complex environments. Episodic memory is a kind of memory that retain human experiences in a particular and conscious way.

In this paper, we propose a self-organizing adaptive recurrent incremental network, termed as SOARIN for spatial map learning. SOARIN is formed by multiple adaptive recurrent Growing When Required network (ar-GWR). The ar-GWR is an extension of Gamma-GWR [8] which consists of a selfadaptive threshold strategy to dynamically adjust the similarity threshold for learning novel sensory information. With the selfadaptive threshold, SOARIN can self-organize neurons according to sensory information themselves. Secondly, we integrate both active sensor and proprioceptive indications (odometry) to train the SOARIN for spatial map building. The integration of both type of sensors can compensate their limitations respectively. The proposed method continually learns spatio-temporal representations from both types sensors and generate a spatial map that can be used for robot localization. We implemented the proposed method to a mobile robot and conducted a series of experiments for evaluation and validation.

The paper is organized as follows. Section II introduces the mathematical models of the proposed method. The experimental setup and results are showed and discussed in Section III and Section IV. Finally, we conclude the paper and highlight some future works.

\section{PROPOSED METHOD: SOARIN}

The proposed method, SOARIN consists of multiple adaptive recurrent GWR networks (ar-GWR) that connected hierarchically. SOARIN models two main memory system which is the working memory and episodic memory for continuous learning. In working memory layer, the ar-GWR learns and encodes compact representations of incoming data samples by adding neurons to the layer. In episodic memory layer, the arGWR quickly learns the sequence of past experiences (events) and encodes the spatiotemporal relations among experiences from working memory layer.

Both networks in the respective layer generate new neurons and update network plasticity intensity according to new sensory input. The learning process in both memory layers is purely unsupervised. In order to strengthen the learned knowledge over time without input information, ar-GWR first reactivates neurons in the episodic memory layer, then selfgenerates neurons activation patterns regularly and replay to the network itself. Thus, SOARIN overcomes the catastrophic forgetting problem for continuous learning tasks.

Episodic neurons are connected to each other and form a spatial map gradually. Each episodic neuron represents a landmark of the explored environment and contains a location and neuron weights which helps robots for localization. When the robot revisits the place, self-localization can be performed by comparing current sensory information with all neurons in the spatial map to determine the winner episodic neuron. If the winner episodic neuron's weight matched with the robot current sensory data, it means that the robot recall and remember that it has been here before and thus retrieved the winner episodic neuron's encoded location for localization.

\section{A. Spatial Map Learning \& Generation}

The episodic memory layer comprises an adaptive recurrent Growing When Required (ar-GWR) self-organizing network that learns the spatiotemporal relationship of input features by incrementally generates neurons and topological connections in the layer. Unlike conventional self-organizing models with winner-take-all rules for spatiotemporal learning, the ar-GWR determines the winner neuron by measuring the neuron activation value based on the current input and a temporal connection. The temporal connection is the sequential relationship of previously activated neurons. As such, each neuron of the layer consists of a weight vector $w_{j}$ and a number $K$ of temporal attributes $e_{j}^{k}$. Thus, neurons in the layer have the recurrent property that will encode sequential activation patterns of the input.

The ar-GWR algorithm consists of 3 main processes: Neuron Activation, Neuron Matching, and Neuron Learning. The network begins with 2 recurrent neurons then undergo the Neuron Activation to determine the best-matching neuron (BMN) $w_{b}$ according to the input $x(t)$ as below:

$$
b=\arg \min \left(T_{j}\right),
$$

$$
\begin{gathered}
T_{j}=\alpha_{1}\left\|x(t)-w_{j}\right\|^{2}+\sum_{k=1}^{K} \alpha_{k}\left\|E_{k}(t)-e_{k, j}(t)\right\|^{2} \\
E_{k}(t)=\beta \cdot w_{J-1}+(1-\beta) \cdot e_{k-1, J-1}
\end{gathered}
$$

where $\alpha_{i}$ and $\beta$ are contributing factors that regulate the influence of the current input according to previously activated neurons, $w_{J-1}$ is the weight of the previous winner neuron 


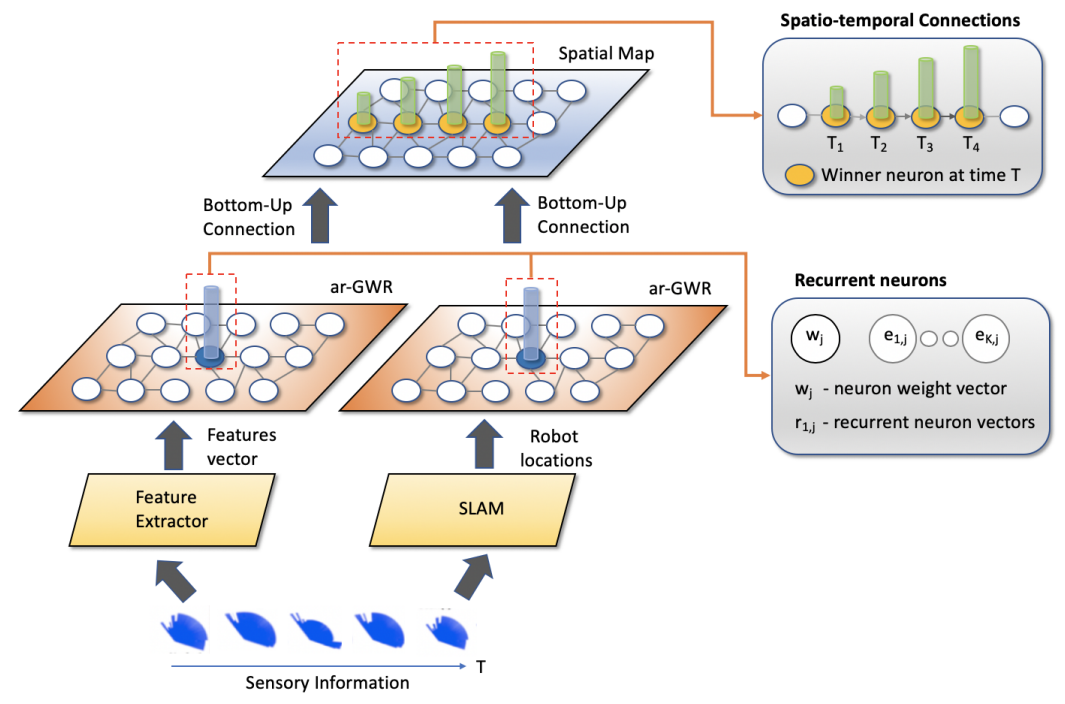

Fig. 1: The SOARIN architecture

at $t-1$, and $E_{k}$ is the global element of the network with $E_{k}(t=0)=0$.

New edges are created between the BMN and the second BMN of a given input. When a BMN is calculated, all the neurons that connected to the BMN are considered as its topological neighbors. When input $x(t)$ is presented, the activation value of the BMN in the network is computed as $a_{b}(t)=\exp \left(T_{b}\right)$ where $T_{b}$ is determined by Eq. 1 to 3 .

Each neuron contains a regularity counter $r_{j} \in[0,1]$ indicating its firing strength over time based on the model stated in [9]. The regularity strength is defined as:

$$
\Delta r_{j}=\tau_{j} \cdot \lambda \cdot\left(1-r_{j}\right)-\tau_{j}
$$

where $\tau_{j}$ and $\lambda$ are decay factors that control the decaying form of the regularity counter [10].

The regularity counter for each newly generated neuron has a value of $r_{j}=1$ and decays towards 0 iteratively. If the neuron's regularity counter $r_{j}$ is smaller than a given threshold $\rho_{r}$, which means the particular neuron is regularized.

At each learning step, a new neuron is added to the network if the regularized BMN neuron activation value $a_{b}(t)$ is smaller than a given threshold $\rho_{a}$ (Neuron Matching). Meaning that a new neuron $N$ is generated if $a_{b}(t)<\rho_{a}$ and $r_{b}<\rho_{r}$ with new weight vectors:

$$
\begin{gathered}
w_{N}=0.5 \cdot\left(x(t)+w_{b}\right), \\
e_{k, N}=0.5 \cdot\left(E_{k}(t)+e_{k, b}\right)
\end{gathered}
$$

For better understanding, the weight vectors and temporal elements in each memory layer is expressed as $w_{j}$ and $e_{k, j}$ respectively. If the BMN neuron is not regularized and $a_{b}(t)>$ $\rho_{a}$, Neuron Learning is performed by updating the BMN $b$ and its topological neighbors $n$ as follows:

$$
\begin{gathered}
w_{j(\text { new })}=\gamma_{j} \cdot r_{j} \cdot\left(x(t)-w_{j(\text { old })}\right), \\
e_{k \text { (new) }}=\gamma_{j} \cdot r_{j} \cdot\left(E_{k}(t)-e_{k, j \text { (old })}\right)
\end{gathered}
$$

where $\gamma_{j}$ is a learning parameter and $\gamma_{n}$ must always smaller than $\gamma_{b}$.

The learning manner of ar-GWR is purely unsupervised and triggered by bottom-up sensory perceptions. The network iteratively accommodates the incoming input data by updating existing neurons or adding new ones. As such, the episodic memory network forms a compact and accommodated representation of the sensory data through self-learning experience.

\section{B. Self Adapting Learning Threshold}

The ar-GWR is an extension of Gamma-GWR where we introduce a self-update threshold for learning instead of using a fixed one. The update equation as follows:

$$
\rho_{(\text {new })}=\left(1-r_{b}\right) \cdot a_{b}(t)+r_{b} \cdot \rho_{(\text {old })}
$$

The self-update equation adjusts the threshold value that closes to the winner BMN activation value if the BMN go through the neuron learning process. This means that the self-update equation lowers the threshold value for learning. This is because the robot observes the environment sequentially and when the BMN go through neuron learning, it means that the network is able to recognize the incoming sensory data and lower the threshold allows the existing network to learn the observed information without adding a new neuron. Thus, it helps to overcome the node proliferation issue. If the BMN does not fulfill the adjusted threshold, a new neuron is added to the network and the threshold is reset to the preset value. 
With the self-update threshold, ar-GWR tends to develop a topological network with lower quantization error of input sequences (shown in Section III). Moreover, ar-GWR generates lesser neurons than Gamma-GWR and requires fewer storage resources for representing input data. This is crucial especially when robot dealing with lifelong learning tasks.

\section{Episodic Layer with Neuron Temporal Connections}

In episodic memory structure, a sequence of events forms an episode to store specific past experiences and episodes are correlating to each other. To emulate the properties of episodic memory, we implement temporal connections that learn activation patterns of recurrent neurons in the network.

The temporal connections encode the sequence of neurons that have been activated during the learning stage. For each learning iteration, a temporal link will be increased by 1 between two neurons that are sequentially stimulated. Specifically, when BMN $b$ and $J-1$ that triggered at time $t$ and $t-1$ subsequently, the temporal link between them is reinforced as follow:

$$
P_{(b, J-1)}^{\text {new }}=P_{(b, J-1)}^{\text {old }}+1,
$$

In this way, for each recurrent neuron $m$, the next neuron $g$ can be retrieved from the encoded temporal sequence by choosing the largest value of $P$ as below:

$$
g=\arg \max P_{(m, n)}
$$

where $n$ are the neighbors of $m$. As a result, the activation sequence of recurrent neurons can be restored without requiring any input data.

\section{Episodic Memory Playback}

According to [11], [12], hippocampal learned knowledge playback is crucial for memory encoding, consolidation, and retrieval. The playback is a process to reactivate memory events without any external cue during both stages of awake and sleep conditions [13].

Hippocampal playback gradually integrates knowledge and experience into more consolidate neural structures via the playback of recently learned knowledge integrated with the currently acquired experiences. Thus, memory reply can mitigate catastrophic forgetting which is a crucial problem of incremental learning tasks.

In this work, randomly feed previous data representations from a generative model or a given sample to the network is not suitable because the temporal relationship of inputs has to be taken into account. In order to generate meaningful sequential data for the playback purpose, we utilize the spatiotemporal relationship of neurons that encoded in the episodic memory layer. The sequential data playback can be generated in episodic memory layer for each episodic neuron whenever the network receives incoming sensory data. For example, if the winner episodic neuron $b$ is triggered by input data, we can determine the next temporal neuron by choosing the neuron that has the largest activation value of $P$ (Section ). A set of neurons playback with length $K^{\mathrm{sm}}+K^{\mathrm{em}}+1$ for each neuron $j$ is computed as follows:

$$
\begin{gathered}
U_{j}=\left\langle w_{u(0)}^{\mathrm{em}}, w_{u(1)}^{\mathrm{em}}, \cdots, w_{u\left(K^{s}\right)}^{\mathrm{em}},\right\rangle \\
u(i)=\arg \max P_{(j, u(i-1))}
\end{gathered}
$$

where $P(i, j)$ is the episodic temporal connection matrix (refer to Section II-C) and $s(0)=j$. Therefore, the temporal connection of episodic neurons that encoded in the network able to generate a sequence of events automatically and replay to the network without additionally storing the relations of previously received training data.

\section{EXPERIMENTAL SETUP \& RESUlTS}

We validate our proposed method using an iRobot Roomba robot that attached with a Hokuyo Laser scanner and Intel i5 processor computer as shown in Figure 2. The laser scanner signal was sampled at $10 \mathrm{~Hz}$. Since the robot has to traverse the environment autonomously, we developed a Fuzzy motion movement behavior that allows the robot for obstacle avoiding and wall-following. The moving speed of the robot varies from $0.05 \mathrm{~m} / \mathrm{s}$ to $0.5 \mathrm{~m} / \mathrm{s}$. As mentioned previous section, the training of network for spatial map building requires both external sensors and proprioceptive indications. In this experiment, we implemented our previous SLAM work [14] to obtain robot location for the SOARIN training. Thus, the network receives a 256 distance features from laser scan and 3 dimensions of robot location (x,y, and $\theta$ ) with a temporal resolution of 3 scans $\left(K^{\mathrm{em}}=2\right)$ for spatial map building. The experiments were conducted in the university corridor, study area, and rest area that connecting with each other. The environment conditions are dynamically populated with pedestrians, changes of lightning state and relocation of university facilities such as tables and chairs. The grid map of the experimental place as shown in Figure 3. We conduct the experiment in such environmental conditions is to validate our proposed method is able to work in natural environment with moderate changing of environmental conditions.

We commanded the robot to traverse the experimental place starting from the study area and travel to the rest area through the corridor then back to the start point again. Each traverse takes about 20 minutes and robot will go to the charging station for recharging. During traverse, SOARIN continually learns incoming sensory information and generates the spatial map for representing the environment. For charging session, memory replay is activated for spatial map memory consolidation. Once robot is fully charged, memory replay is deactivated and robot starts traversing and continue learns and updates the spatial map again. We repeated the experiment for ten times and the generated spatial map quality was measured by Total Quantization Error (TQE) and localization rate. Figure 4 shows the spatial map after the last traverse and each episodic neuron is learned and activated by a temporal resolution of input data. Figure 5 illustrates the total number of neurons in the map that generated by SOARIN and [8] for each traverse. TQE measures the similarity between sensory information and weights of episodic neurons in the spatial map. Figure 6 shows 


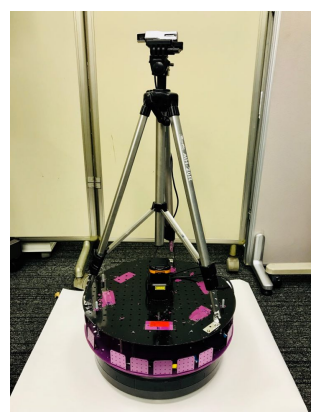

Fig. 2: Robot attached with laser scanner

TABLE I: Parameter settings

\begin{tabular}{|c||c|}
\hline Parameter & Value \\
\hline$\alpha^{1}, \alpha^{2}$ & 0.5 \\
$\rho_{a}$ & 0.75 \\
$\rho_{r}$ & 0.1 \\
$\tau_{j}$ & 0.5 \\
$\lambda$ & 1.05 \\
$\beta$ & 0.5 \\
$\gamma_{b}$ & 0.2 \\
$\gamma_{n}$ & 0.001 \\
$r_{e}$ & 0.001 \\
\hline
\end{tabular}
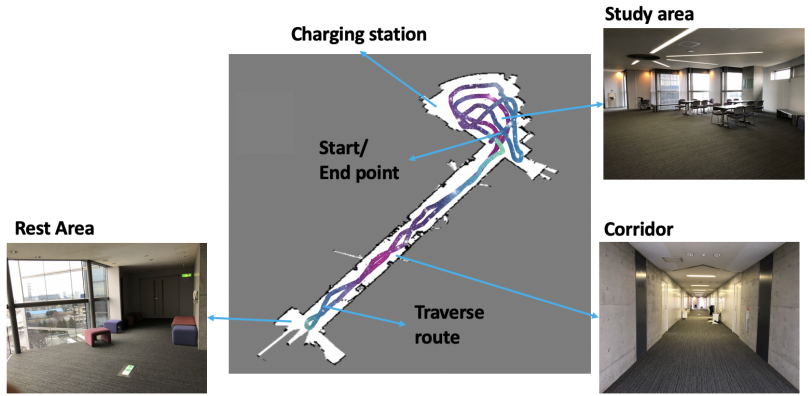

Fig. 3: Grid map of corridor, study area and rest area

the TQE of the learning approach for each traverse. Next, the localization rate is measured by computing the Euclidean distance between the winner neuron's encoded location and robot current location that obtained from the SLAM algorithm. If the Euclidean distance is less than a preset value $(0.01 \mathrm{~m})$, the robot is localized successfully. The localization rate result is shown in Figure 7.

The parameters setting of the proposed method for spatial map building as tabulated in Table I.

\section{Discussion}

SOARIN framework is formed by multiple adaptive recurrent GWR (ar-GWR) networks hierarchically that continually learns the spatiotemporal relationship of sensory information. SOARIN is the extension of Gamma-GWR [8] that learns spatiotemporal has a self-adaptive threshold mechanism. In this

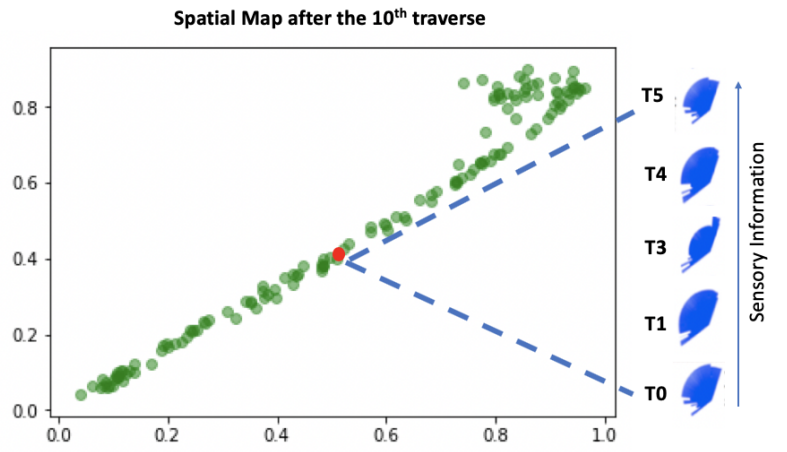

Fig. 4: Spatial map that generated by SOARIN

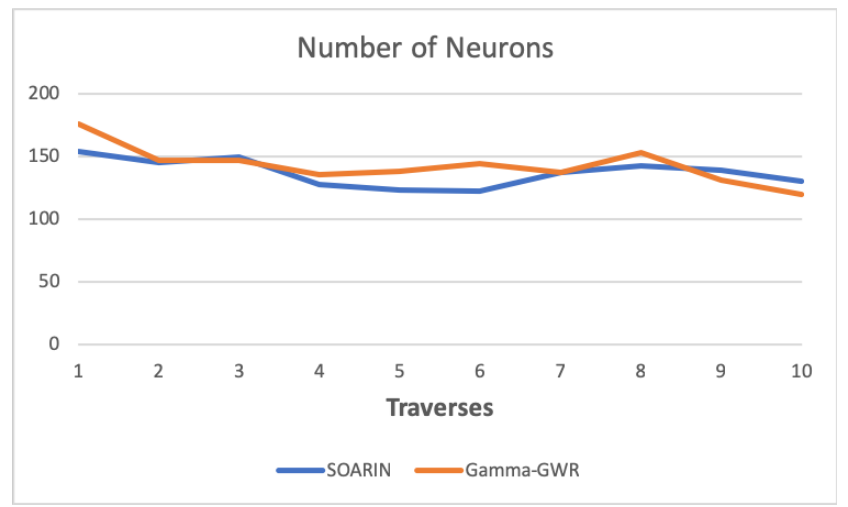

Fig. 5: Total number of generated neurons for each traverse

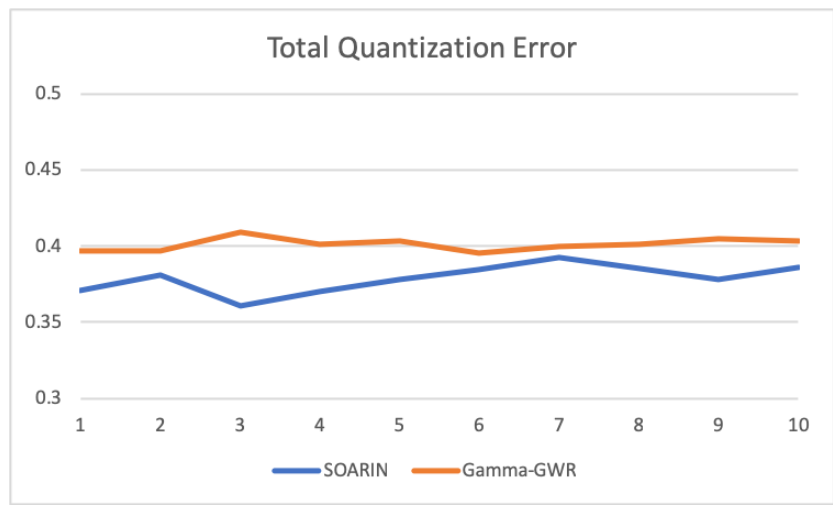

Fig. 6: Total Quantization Error of the learning method for each traverse

paper, SOARIN learns sensory information from both active sensors and proprioceptive indicators that compensate each sensor limitations for incrementally generates a spatial map. The spatial map is used for robot localization without human predefined knowledge. The performance of SOARIN has been validated and compared with the state-of-art method [8] through real robot experiments. Results showed that the lo- 


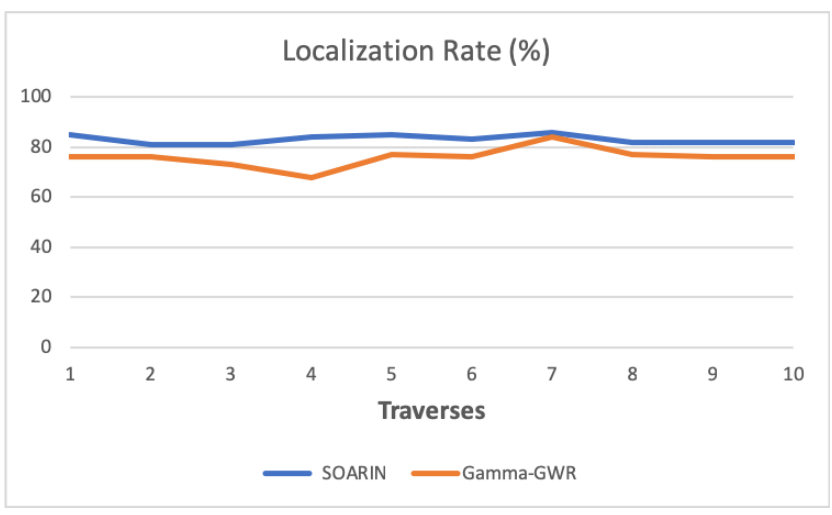

Fig. 7: Localization rate of the learning method for each traverse

calization rate is better than [8] and yielded to slightly lower total quantization error as well. The average localization rate of SOARIN is $83.1 \%$ which represents an increase of $7.2 \%$ with respect to the [8] approach. The failure of localization happens when the robot encounters a sudden change of the environment for instance pedestrian suddenly step out from the laboratory during robot navigation. In addition, the number of neurons of the ten traverse is within 122 and 154 neurons which showed that SOARIN able to expand and shrink the spatial map structure depending on the environmental conditions. This is one of the significant improvement of our previous work [15] which the episodic network structure is continually growing as time goes by that leads to node proliferation issues for long time operation.

\section{CONCLUSION}

In this paper, we proposed a self-organizing network termed as SOARIN that model human episodic memory which can continually learn the spatiotemporal relationship of sensory data from both active sensors and proprioceptive indications to generate a spatial map incrementally. SOARIN updates the spatial map by expanding or shrinking its episodic memory structure autonomously. In addition, SOARIN consolidates the spatial map through its self episodic memory playback without the needs of external sensory cues. SOARIN has been validated through real robot implementation. In the future, we will integrate SOARIN with path planning algorithm to utilize the topological structure of the spatial map for goal-directed navigation. Lastly, we will further improve and validate the performance of SOARIN in more challenging and larger environments.

\section{ACKNOWLEDGMENT}

The authors would like to thank Tokyo Metropolitan Government for supporting the research by providing the Human Resources Fund.

\section{REFERENCES}

[1] J. O'Keefe and L. Nadel, "The hippocampus as a cognitive map," Behavioral and Brain Sciences, vol. 2, no. 4, p. 487494, 1979.

[2] S. Thrun, D. Fox, W. Burgard, and D. F., "Robust monte carlo localization for mobile robots," Artificial Intelligence, vol. 128, no. 1-2, 2001.

[3] S. Thrun, "Learning metric-topological maps for indoor mobile robot navigation," Artif. Intell., vol. 99, no. 1, pp. 21-71, Feb. 1998.

[4] W. H. Chin, C. K. Loo, M. Seera, N. Kubota, and Y. Toda, "Multi-channel bayesian adaptive resonance associate memory for on-line topological map building," Applied Soft Computing, vol. 38, pp. 269 - 280, 2016. [Online]. Available: http://www.sciencedirect.com/science/article/pii/S1568494615006031

[5] D. Ball, S. Heath, J. Wiles, G. Wyeth, P. Corke, and M. Milford, "Openratslam: An open source brain-based slam system," Auton. Robots, vol. 34, no. 3, pp. 149-176, Apr. 2013. [Online]. Available: http://dx.doi.org/10.1007/s10514-012-9317-9

[6] U. M Erdem and M. Hasselmo, "A biologically inspired hierarchical goal directed navigation model," Journal of physiology, Paris, vol. 108, 072013.

[7] Y. Endo, "Anticipatory robot control for a partially observable environment using episodic memories," in 2008 IEEE International Conference on Robotics and Automation, May 2008, pp. 2852-2859.

[8] G. I. Parisi, J. Tani, C. Weber, and S. Wermter, "Lifelong learning of human actions with deep neural network self-organization," Neural Networks, vol. 96, pp. 137 - 149, 2017. [Online]. Available: http://www.sciencedirect.com/science/article/pii/S0893608017302034

[9] J. C. STANLEY, "Computer simulation of a model of habituation," Nature, vol. 261, pp. 146-8, 061976.

[10] S. Marsland, J. Shapiro, and U. Nehmzow, "A selforganising network that grows when required," Neural Networks, vol. 15 , no. 8, pp. 1041 - 1058, 2002. [Online]. Available: http://www.sciencedirect.com/science/article/pii/S0893608002000783

[11] D. Kumaran, A. Banino, C. Blundell, D. Hassabis, and P. Dayan, "Computations underlying social hierarchy learning: Distinct neural mechanisms for updating and representing self-relevant information," Neuron, vol. 92, no. 5, pp. 1135 - 1147, 2016. [Online]. Available: http://www.sciencedirect.com/science/article/pii/S0896627316308029

[12] M. F. Carr, S. P. Jadhav, and L. M. Frank, "Hippocampal replay in the awake state: a potential substrate for memory consolidation and retrieval," Nature Neuroscience, vol. 14, pp. 147 EP -, 012011. [Online]. Available: https://doi.org/10.1038/nn.2732

[13] D. C. McClelland, "Achievement motivation in relation to achievementrelated recall, performance, and urine flow, a marker associated with release of vasopressin," Motivation and Emotion, vol. 19, pp. 59-76, 031995 .

[14] Y. Toda and N. Kubota, "Self-localization based on multiresolution map for remote control of multiple mobile robots," IEEE Transactions on Industrial Informatics, vol. 9, no. 3, pp. 1772-1781, Aug 2013.

[15] W. H. Chin, Y. Toda, N. Kubota, C. K. Loo, and M. Seera, "Episodic memory multimodal learning for robot sensorimotor map building and navigation," IEEE Transactions on Cognitive and Developmental Systems, pp. 1-1, 2018. 